\title{
Long-term solar activity and terrestrial connections." Part I: theory
}

\author{
N. D. Diamantides \\ Department of Geography, Kent State University, P.O. Box 5190, Kent, Ohio 44242, USA
}

Received: 25 March 1996 / Revised: 16 August 1997 / Accepted: 5 September 1997

\begin{abstract}
The research task described herein aims at the structuring of an analytical tool that traces the time course of geophysical phenomena, regional or global, and compares it to the course of long-term solar conditions, long-term meaning decades or a few centuries. The model is based on the premise that since in a last analysis the preponderance of atmospheric, hydrospheric, and, possibly, some aspects of geospheric phenomena are, or have been, powered by energy issuing from the sun - either now or in the past, the long-term behavior of such phenomena is ultimately "connected" to long-term changes occurring in the sun itself. Accordingly, the proposed research firstly derives and models a stable surrogate pattern for the long-term solar activity, secondly introduces a transfer-function algorithm for modeling the connection between the surrogate and terrestrial phenomena viewed as partners in the connection, and thirdly probes the connection outcome for episodic or unanticipated effects that may arise due to the fact that in the present context, the connection, should it exist, is very likely nonlinear. Part I of the study presents the theory of the concept, while Part II demonstrates the concept's pertinence to a number of terrestrial phenomena.
\end{abstract}

Key words. Solar activity $\cdot$ Kolmogorov algorithm

\section{Introduction}

The particular aim of this essay is to examine the macrodynamic aspects of solar activity without invoking the particular agents that produce it (such as plasma

\footnotetext{
* This essay has been the basis of two earlier papers, one presented at "The 8th International Conference of the European Union of Geosciences" in Strasbourg, France, 9-13 April 1995; and the other at "The 5th International Conference on Precipitation" in Elounda, Crete, Greece, 14-16 June 1995.
}

physics, helioseismology, planet-induced tidal effects, etc), and to do this in view of the presently incomplete knowledge of the actual mechanisms involved. Consequently, the outcome of this research will be descriptive of behavior morphology, and not necessarily explanatory of causation as far as the phenomena in question are concerned. In this context, let it be remembered that Isaac Newton is often cited in support of the school which prefers to establish measurements rather than to ascertain causes, "hypotheses non fingo", as he said.

However, he was far from suggesting this as a limitation of scientific research in general, as the following passage from his Opticks attests:

To derive two or three Principles of Motion ...would be a very great step in Philosophy, though the Causes of those Principles were not yet discover'd: And therefore I scruple not to propose the Principles of Motion abovemention'd, they being of very general Extent, and leave their causes to be found out. (Samuel, 1952).

Our probing is based on the premise that since in a last analysis the preponderance of atmospheric, hydrospheric, and possibly some aspects of geospheric phenomena are or have been powered by energy issuing from the sun, their long-term behavior reflects long-term changes occurring in the sun itself. Hence, the proposed research

1. proposes and models a stable surrogate pattern for the long-term solar activity;

2. introduces a transfer-function algorithm for modeling the connection between this surrogate and terrestrial phenomena for which solar activity has relevance;

3. probes the connection outcome for episodic or unanticipated events, given that the connection, should it exist, is very likely nonlinear.

\section{Methodology}

It must be stated from the beginning that the basic nature of the analysis attempted here is purposely cross- 
disciplinary, partaking of both physical science and engineering. This stems from the conviction that theoretical tools which sent man to the moon and are now preparing equipment for the unmanned exploration of Mars, can also assist geoscience in deciphering messages from the sun, should they exist. However, regarding solarterrestrial connections, such geophysically basic occurrences as the El Niño in the Pacific, the temperature of either hemisphere, volcanos throughout the globe, and tree-rings in different locales, are occurrences which hardly interest most engineering analysts. By the same token, such potent tools of the latter as transfer-functions and system-response algorithms that deal with dynamic phenomena in general, do not people the tool kit of geophysisists at large. Against this background, our study takes the position that in order to proceed intelligently with regard to solar-terrestrial connections, the two types of skill need to complement each other on the task difficulties notwithstanding (Keyfitz, 1992, 1994) - and that the Kolmogorov algorithm is the medium that can bind the two together in the present context: its principal worth being that it implicitly captures the dynamics propelling a given phenomenon from the phenomenon's past course, even where no adequate theoretical knowledge of such dynamics presently exists. The algorithm's detailed derivation in Part I is for the benefit of the geoscience element of the interdisciplinary team.

The analysis begins with the observation that solar activity is not only in tune with the familiar 11-year cycle of the sunspot number, but, in addition, varies along a time-scale measured in centuries, as the Sporer and Maunder Minima demonstrate (Eddy, 1976; Hughes, 1977). One approach to examining the activity's longterm behavior will be to consider the sunspot number as its surrogate and, through it, structure a mathematical model of solar activity potentially useful to the study of whatever phenomena such activity may have a bearing upon.

The suggested method proceeds with the task of modeling the sunspot number in the long run based on the following tenet and its corollary:

The Constrain Tenet. Any solar process involving the exchange or release of energy $y(t)$, has an upper bound $Y$, and a time rate of change $\mathrm{d} y / \mathrm{d} t$ that increases with both the current value of $y(t)$ and the remaining potential $Y-y(t)$ for further change. This renders both $y$ and $\mathrm{d} y / \mathrm{d} t$ bounded. The modality is not new, having been introduced by P. F. Verhulst in 1844 in connection with population growth (Lotka, 1956).

The Corollary. Since the constrain tenet means that the process $y(t)$ is nonlinear, the process cannot only give rise to a basic oscillation $f_{0}$, but also cause:

1. the appearance of new incommensurable frequences $f_{1}, f_{2}, \ldots$,

2. the entrainment of these frequencies so that (for example) $f_{1}, f_{2}$ approach values $f_{0}$,

3. the sudden appearance of aperiodic spectra,

4. the appearance of subharmonics $f_{1} / 2, f_{2} / 4$ successively (McDonald, 1976).
Put in terms of a differential equation, the tenet requires that the process evolves as:

$\frac{\mathrm{d} y}{\mathrm{~d} t}=c y(Y-y)$,

where $c=c(t)$ is a factor, facilitative or inhibitory, established by the determinants of the process in question, which therefore may be called the mediating factor. The expression in Eq. (1) is a Bernoulli-type differential equation when $c$ is time dependent, and becomes the well-known logistic law when $c$ is constant. The equation's general solution is:

$$
y(t)=\frac{1}{\left(1 / y_{\min }-1 / y_{\max }\right) \exp \left(-y_{\max } \int c(t) \mathrm{d} t\right)+1 / y_{\max }},
$$

and

$$
\int c(t) \mathrm{d} t=\ln \frac{1 / y_{\min }-1 / y_{\max }}{1 / y(t)-1 / y_{\max }} .
$$

Depending on $c(t)$, this basic expression can model a great variety of physical (as well as societal) phenomena and, being nonlinear, can lead to outcomes of unexpected complexity, including cases of the so-called deterministic chaos (May, 1976; Modis and Bebecker, 1992; Mullin and Price, 1989). The basic features of Eq. (1) are that:

1. $\mathrm{d} y / \mathrm{d} t=0$ when $y=0$ or $y=Y$,

2. $\mathrm{d} y / \mathrm{d} t=\max$ when $2 y=Y-1 / c^{2} \mathrm{~d} c / \mathrm{d} t$.

To compute the two extremes $y_{\min }$ and $y_{\max }$ for Eq. (2) from the data of a particular case, we may use the expedient of approximating either end $y_{\text {end }}$ by an exponential curve; it is easy to show that three equidistant points of such a curve will produce:

$y_{\text {min }}$ or $y_{\max }=\frac{y_{\mathrm{end}}(t)^{2}-y_{\mathrm{end}}(t-a) y_{\mathrm{end}}(t+a)}{2 y_{\mathrm{end}}(t)-y_{\mathrm{end}}(t-a)-y_{\mathrm{end}}(t+a)}$.

The two features in Eq. (3) imply a cusp-like shape for $\mathrm{d} y / \mathrm{d} t$, simple when $c(t)=c$ (constant), and more complicated when $c(t)$ varies in time. When, as in the present context, the mediating factor is a priori unknown, the morphology of $\mathrm{d} y / \mathrm{d} t$ may be approximated by the expression:

$\frac{\mathrm{d} y}{\mathrm{~d} t}=\exp \left(a t^{2}+b t+k\right), \quad a<0, \quad a, b, k=$ const.

as an intermediate step. This simple function is chosen because it allows for a single peak only and, therefore, for the capture of the very core of the growth process. The condition upon the parameter $a$ ensures that in the limit $\mathrm{d} y / \mathrm{d} t$ settles at a finite nonnegative level, as it should for the constrain tenet to be satisfied. Given that the root of the derivative of $\mathrm{d} y / \mathrm{d} t$ in Eq. (5) defines the time $t_{p}$ of the $\mathrm{d} y / \mathrm{d} t$ peak as $t_{p}=-b / 2 a$, it follows that when $t_{p}$ is known, Eq. (5) becomes: 
$\frac{\mathrm{d} y}{\mathrm{~d} t}=\exp \left[a t\left(t-2 t_{p}\right)+k\right]$.

Since the parameters $a, k$ in Eq. (6) can be found from the data, $y(t)$ and $Y$ are computable using Eq. (1) and, through them, the mediating factor $c(t)$.

\section{Model synthesis}

The long-term behavior of solar activity can be brought into focus by filtering out the ubiquitous annual mean relative Wolf sunspot number and its 11-year periodicity.

The simplest means of effecting this is through the 11year moving average (Eddy, 1976), for which the symbol $z_{11}(t)$ will be used:

$z_{11}(t)=\frac{1}{11} \sum_{t-10}^{0} z(t)$,

the time history of which may be seen in Fig. 1.

The $z(t)$ data on which the present analysis is based comes from McKinnon (1987) for the years 1700-1985, and from the monthly issues of $S k y$ and telescope after 1985. It should be remarked that the reliability of the data deteriorates as one goes back in time (Eddy and Stuiver, 1977), but they are deemed accurate enough at least from 1800 to the present: the assessment is in agreement with the results of a study by Hoyt et al. (1994) who used 340000 daily sunspot group counts from more than 350 observers active between 1610 and 1993.

Three prominent features characterize $z_{11}(t)$ over the course of the almost three hundred years for which data exist:

1. a continuously rising secular trend $T(t)$,

2. long-term surging $S(t)$,

3. a quasi-patterned transient $\operatorname{Tr}(t)$.

Among them, $T(t)$ and $S(t)$ constitute the steady-state part of $z_{11}(t)$. Of these two, the $S(t)$ part gives a strong indication of the 'Gleissberg Cycle' (Eddy, 1976), represented by approximately two-and-a-half surges in

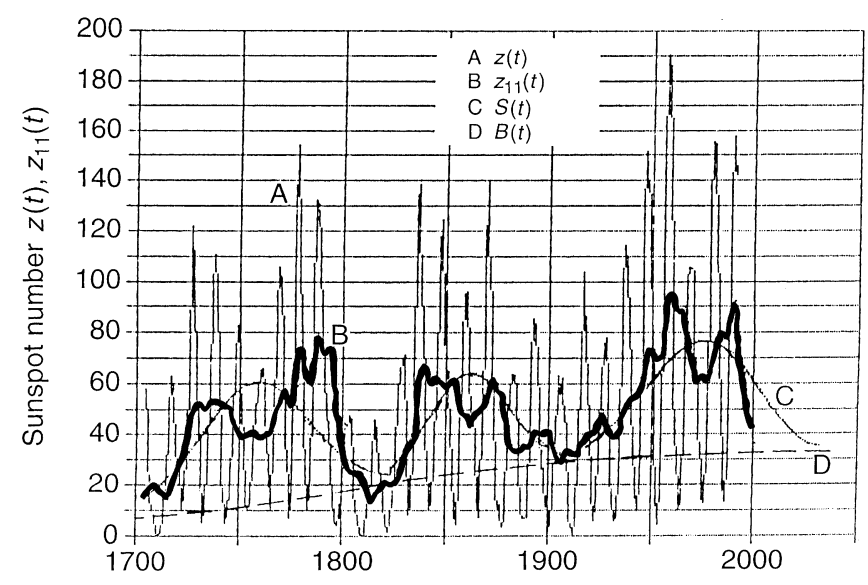

Fig. 1. Sunspot short-term and long-term the spans 1705-1811, 1812-1905, and 1906-?. Quite obviously, the course of each surge is of the type implied by Eq. (1). All three surges ride over what may be called a base-line which parallels the secular trend, and all three feature a definite bifurcation in the neighborhood of the surge peak. In addition, a higher-frequency transient $\operatorname{Tr}(t)$ is obvious in all three surge cases, causing a pair of subpeaks for each surge.

\subsection{Secular trend $T(t)$}

While the upward trend of $z_{11}(t)$ over the last three centuries is quite obvious in Fig. 1, its increase with time cannot be open ended if the constrain tenet is to be satisfied. However, since no peaking of $T(t)$ has been discerned over the length of the record, the analysis has to assume such a peaking at some future time $t_{p}$ and, based on it, determine the course of $T(t)$ using Eq. (6) in the form:

$\ln \frac{\mathrm{d} T(t)}{\mathrm{d} t}=a t\left(t-2 t_{p}\right)$.

The $t_{p}$ value can be found by applying linear regression iteratively to the 1700-1991 record and selecting the $t_{p}$ that minimizes the goodness-of-fit coefficient $R^{2}$. The result, including year of the peak $t_{p}$, peak value $T_{p}$, and duration $t_{d}$, is given by:

$a=-0.410 \times 10^{-6}\left(0.031 \times 10^{-6}\right)$,

$k=3.41$,

$t_{p}=2100 \mathrm{AD}$,

$T_{p}=60.8$,

$t_{d}=800$ years .

If, in violation of the constrain-tenet, the foregoing parameters were computed without imposing a peaking requirement, the result would be:

$a=0.800 \times 10^{-5}\left(0.064 \times 10^{-5}\right)$,

$b=-0.383 \times 10^{-4}\left(0.044 \times 10^{-5}\right)$,

$k=3.574$,

$t_{p}=-b / 2 a=2.4$ years.

This implies unbounded growth for $T(t)$, due to the fact that the end of the data record used is the ascending part of the third surge presently at its peak. It is of interest, however, that the curve of Eq. (10) bottoms out in the year 2.4, or $1707 \mathrm{AD}$, which is the ending era of the Maunder Minimum.

For the base line $B(t)$, on the basis of which the surges are to be computed, the following simple definition was chosen:

$$
\begin{aligned}
B(t) & =T(t)-B \\
B & =24.1 .
\end{aligned}
$$

This is simply the bounded curve of Eq. (9) lowered by $k$ units of $z_{11}(t)$ to between the 1815 and 1906 minima of $z_{11}(t)$. 
Table 1. Solar activity periods

\begin{tabular}{llc}
\hline name of maximum & timing & $\begin{array}{l}\text { time between } \\
\text { maxima (years) }\end{array}$ \\
\hline Homeric & $1375-700 \mathrm{BC}$ & 675 \\
Greek & $700-375 \mathrm{BC}$ & 325 \\
Roman & $375 \mathrm{BC}-690 \mathrm{AD}$ & 1065 \\
Medieval & $690-1490 \mathrm{AD}$ & 800 \\
Columbian & $1490-1675 \mathrm{AD}$ & 185 \\
Modern & $1675-2475 \mathrm{AD}$ & $800(?)$ \\
\hline
\end{tabular}

The duration of $T(t)$, and therefore $B(t)$, was established by first finding the base line's value $B(1715)=6.64$ at the end of the Maunder Minimum, and computing the future year during which the descending branch of $B(t)$ reaches the same level. To place the result in historical perspective, we observe that the contemporary trend $T(t)$ corresponds to the latest of Eddy's historical 'maxima' of the sunspot activity's envelope using ${ }^{14} C$ as a guide (Stuiver and Quay, 1980). Of these, the last six have occurred in historical times as seen in Table 1, "Columbian" being our designation for the spurt of envelope activity that took place between the Sporer and Maunder Minima. Duration comparison between the two tabulations shows the ongoing Modern Maximum to be on par with the Medieval Maximum.

\subsection{The surges $S(t)$}

The morphology of the surging part of $z_{11}(t)$ may be looked into either collectively or individually.

3.2.1 Collective surging. From a collective viewpoint, particularly dominant over the post-Maunder era appear to be two oscillations with periods of about 179 and 110 years (Williams, 1981). The first oscillation in particular has attracted researchers' attention due to the fact that it approaches the behavior of orbital phenomena in the solar system: Jose (1965) has shown this periodicity to be prominent in the motion of the sun about the center of mass of the solar system, and Gribbin and Plagemann (1974) point out that 179 years is the period of total planetary alignment during which every planet is in conjunction with every other planet on the same side of the sun. Wood (1972), in analyzing the connection between sunspot number and the height of the planetary gravity tide on the sun's surface, observes that "the curve appears to repeat after about 170 or 180 years". Cohen and Lintz (1974), on the other hand, suggest that "although the 179-year periodicity does exist in the sunspot cycle, it is not caused by a long-term planetary excitation but is, instead, a beat phenomenon". By subjecting the 1844-1971 $z(t)$ data to a maximum-entropy spectral analysis, they found three dominant frequencies in the spectrum: 110, 10.9, and 9.7 years. Of these the latter two "interfere to produce beats of roughly 187 years". In our own work we found that the 178.7- and 110-year components do make for a good representation of the long-term surging in $z_{11}(t)$. To arrive at this, the $S(t)$ were separated from $z_{11}(t)-B(t)$ and Eq. (8) was applied for each surge separately, leading to the cusps seen in Fig. 1, and the following parameters:

$\begin{array}{llll}\text { surge } & S_{\text {I }} & S_{\text {II }} & S_{\text {III }} \\ a & -0.0007 & -0.0014 & -0.0006 \\ & (0.00005) & (0.00010) & (0.00004) \\ b & 0.0631 & 0.1349 & 0.0786 \\ k & 2.497 & 0.488 & 1.238 \\ R^{2} & 0.565 & 0.655 & 0.820 \\ \text { area } S_{A} & 3435 & 1890 & 3370 \\ \text { peak } & 62.1 & 62.9 & 78.1 \\ \text { peak year } & 1758 & 1865 & 1973 \\ \text { duration } & 1705-1821 & 1821-1906 & 1906-2045 .\end{array}$

In a follow-up step, the surge function $S_{j}, j=\mathrm{I}$, II, III, were expressed in terms of the aforesaid sinusoids:

$$
\begin{array}{r}
S_{j}(t)=\sum_{n} \sum_{N}\left[a_{n} \sin \left[\frac{2 \pi}{N}(t-1705)\right]\right. \\
\left.\quad+b_{n} \cos \left[\frac{2 \pi}{N}(t-1705)\right]\right]+k_{0} \\
n=1,2, \quad N=178.7,110, \quad 1705 \leqslant t \leqslant 1991,
\end{array}
$$

with the following results:

$$
\begin{aligned}
& a_{1}=4.423(0.313) \\
& b_{1}=-4.559(0.322) \\
& a_{2}=6.334(0.323) \\
& b_{2}=-17.320(0.315), \\
& k_{0}=25.35 \\
& R^{2}=0.93
\end{aligned}
$$

Projecting into the future, this model suggests that the core (not counting the transient) of the next surge $S_{\text {IV }}$ will peak in the year 2090 at the level of 43.9 units above the base line, as Fig. 2 portrays it. This renders the ongoing surge $S_{\text {III }}$ the strongest in the span 1700-2100 of four centuries, with a core peak of 48.3 units above the base line. With the base line included, $S_{\text {III }}$ and $S_{\text {IV }}$ emerge about equal (79.8 units).

3.2.2 Individual surges. While the use of the Eq. (6) expedient serves a purpose in that it gives an overview of the long-term activity, it is not conducive to probing the morphology of the surges' internal structure. For this, not only the core part $S(t)$, but the entire surge $S(t)+\operatorname{Tr}(t)$ needs to be dealt with, i.e.:

$$
S(t)+\operatorname{Tr}(t)=z_{11}(t)-B(t) .
$$




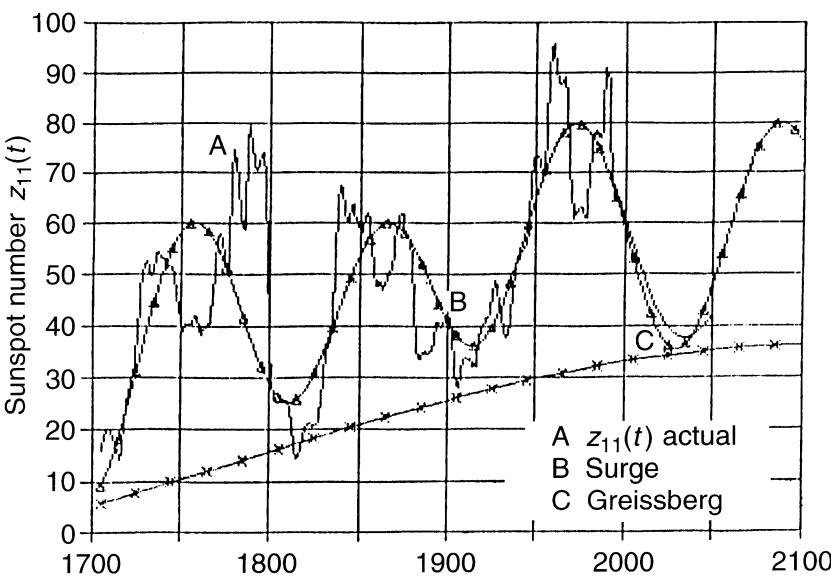

Fig. 2. Collective surging

After finding the total area $S_{A}$ under the curve of the right-hand member, use of it and of $s(t)$ in Eq. (1) produces the mediating factor $c(t)$, that is the key structural element of the model, and the nature of which can be seen in Fig. 3. This is manifestly a trigger type of occurrence whose time behavior is made up of the combination of a growth term and a decay term operating concurrently,

$c_{0}(t)= \begin{cases}c_{0} & \text { if } t=0 \\ c_{1} \mathrm{e}^{+\alpha t}+c_{2} \mathrm{e}^{-\beta t}, & \text { if } t>0\end{cases}$

with the parameter values of each case seen in the following equation and the process pictured in Fig. 4.

$$
\begin{array}{llll}
\text { surge } & S_{\text {I }} & S_{\text {II }} & S_{\text {III }} \\
c_{1} & 0.362 \times 10^{-4} & 1.553 \times 10^{-4}, & 0.351 \times 10^{-4} \\
c_{2} & 0.032 \times 10^{-5} & 0.743 \times 10^{-5}, & 0.104 \times 10^{-5} \\
\alpha & 0.055(0.004) & 0.022(0.002) & 0.029(0.002) \\
\beta & -0.029(-0.002) & -0.041(0.003) & -0.215(0.016
\end{array}
$$

Of interest is the fact that the observed dip at each $S(t)$ peak occurs near the crossing of the two $c_{0}(t)$ branches in Eq. (16). The term $c_{1}$ depicts a positive feedback process, which at some level triggers the negative

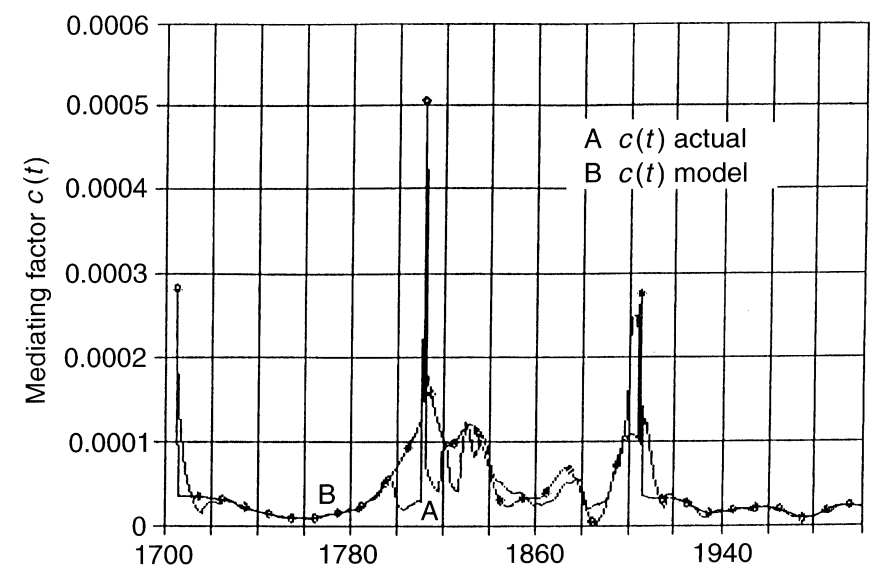

Fig. 3. Mediating factor (actual and model)

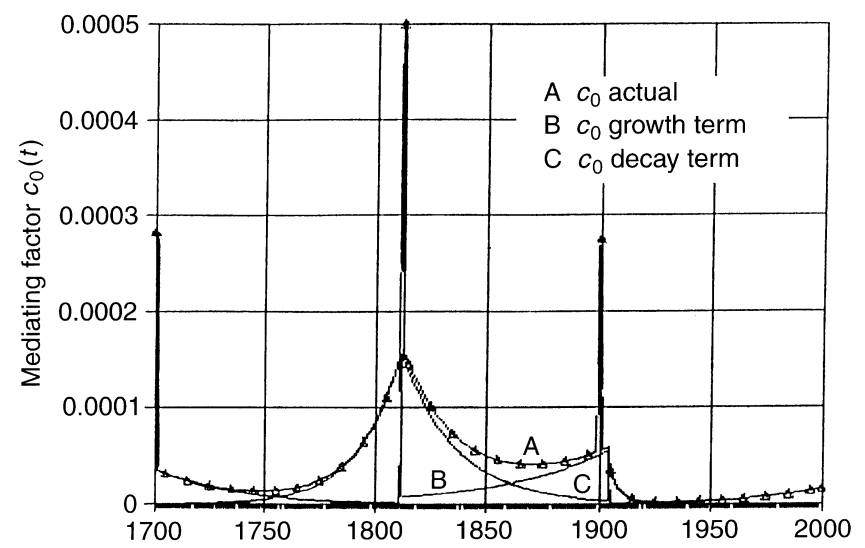

Fig. 4. Mediating factor (growth and decay)

feedback process represented by the negative feedback term $c_{2}$ of the next surge's mediating factor. Interestingly enough, this modality replicates a relationship between solar activity and the earth's magnetic field: the diurnal variation of the horizontal component of this field on abnormally quiet days (AQD) at the time of sunspot minimum consistently anticipates the magnitude of the subsequent sunspot minimum (Brown, 1974). This pattern implies "that the size of solar activity maximum is determined at the very beginning of a cycle, or perhaps the very end of the preceding cycle from the 'depth' of the solar minimum" (Brown, 1974).

With $c_{0}(t)$ known, integration of the expression in Eq. (1) produces the equation $s(t)$ of each individual surge as:

$$
\begin{aligned}
s(t)= & \frac{A S_{A} c_{0}(t) \exp (-J)}{[A \exp (-J)+1]^{2}}, \\
A & =\frac{1}{s_{0}}-\frac{1}{S_{A}}, \\
J & =S_{A}\left[\frac{c_{1}}{\alpha}\left(\mathrm{e}^{+\alpha t}-1\right)-\frac{c_{2}}{\beta}\left(\mathrm{e}^{-\beta t}-1\right)\right]+i_{0},
\end{aligned}
$$

where $s_{0}$ is the value of $S(t)$ at the time $t_{0}$ of the surge onset, and $i_{0}$ the integration constant. The values of these two parameters, along with that of the model $S_{A}$, are given as

$\begin{array}{llll}\text { surge } & S_{\mathrm{I}} & S_{\mathrm{II}} & S_{\mathrm{III}} \\ S_{A} & 3560 & 1990 & 3630 \\ S_{0} & 9.82 & 3.87 & 2.27 \\ i_{0} & 0.002 & 0.003 & 0.002 .\end{array}$

Table 2 lists the projected values of the steady-state part of $z_{11}(t)$ in column $\mathrm{A}$, from the present to the end of surge $S_{\mathrm{III}}$. In column $\mathrm{B}$ of the table an alternate projection is listed, based on the observation that the surge $S_{\text {III }}$ appears to be symmetric about the 1977 position and, therefore:

$$
\begin{aligned}
& S_{\mathrm{III}}(1977+t)=S_{\mathrm{III}}(1977-t)+k, \\
& k=-0.95, \quad t \geqslant 14 .
\end{aligned}
$$


Table 2. $z_{11}(t)$ projection

\begin{tabular}{|c|c|c|c|c|c|}
\hline \multirow[t]{2}{*}{ year } & \multicolumn{2}{|l|}{$z_{11}(t)$} & \multirow[t]{2}{*}{ year } & \multicolumn{2}{|l|}{$z_{11}(t)$} \\
\hline & A & B & & A & B \\
\hline 1990 & 94.5 & & 2007 & 57.9 & 58.6 \\
\hline 1991 & 93.5 & 94.6 & 2008 & 56.4 & 56.3 \\
\hline 1992 & 91.8 & 89.9 & 2009 & 54.9 & 54.1 \\
\hline 1993 & 89.5 & 92.6 & 2010 & 53.5 & 51.9 \\
\hline 1994 & 86.8 & 94.6 & 2011 & 52.1 & 49.9 \\
\hline 1995 & 83.9 & 92.6 & 2012 & 50.8 & 46.4 \\
\hline 1996 & 80.9 & 89.6 & 2013 & 49.9 & 44.9 \\
\hline 1997 & 78.0 & 80.7 & 2014 & 48.3 & 43.7 \\
\hline 1998 & 75.2 & 70.8 & 2015 & 47.2 & 42.8 \\
\hline 1999 & 72.6 & 68.2 & 2016 & 46.2 & 42.1 \\
\hline 2000 & 70.2 & 69.3 & 2017 & 45.3 & 41.6 \\
\hline 2001 & 68.0 & 72.9 & 2018 & 44.5 & 41.3 \\
\hline 2002 & 66.0 & 70.5 & 2019 & 43.8 & 41.2 \\
\hline 2003 & 64.2 & 68.1 & 2020 & 43.2 & 41.1 \\
\hline 2004 & 62.5 & 65.7 & 2025 & 40.7 & 40.9 \\
\hline 2005 & 60.9 & 63.3 & 2030 & 38.4 & 37.5 \\
\hline 2006 & 59.4 & 61.0 & 2035 & 36.6 & 32.8 \\
\hline
\end{tabular}

The restriction upon $t$ places the projection at the year 1992 or later, while the constant $k$ makes the average value of the difference between columns $\mathrm{A}$ and $\mathrm{B}$ equal to zero. It is curve B that will be used in Part II of this study.

The following surge characteristics are presented at this point in order to effect comparisons:

$\begin{array}{lllll} & \text { peak 1 } & \text { trough } & \text { peak 2 } & \text { surge } \\ & \text { position } & \text { position } & \text { position } & \text { duration } \\ S_{\text {I }} & 0.301 & 0.528 & 0.783 & 1(=106 \text { years }) \\ S_{\text {II }} & 0.293 & 0.521 & 0.783 & 1 \text { (= 92 years }) \\ S_{\text {III }} & 0.400 & 0.540 & 0.637 & 1 \text { (=125 years). }\end{array}$

\subsection{The transient $\operatorname{Tr}(t)$}

The relatively shorter period undulations $\operatorname{Tr}(t)$ exhibited by $z_{11}(t)$, in addition to the long-span surges, are accounted for by the basic tenet's corollary and therefore have to be represented by a term $c_{r}(t)$ in the mediating factor $c(t)$. Accordingly, and for each surge separately, the residual:

$c_{r}(t)=c(t)-c_{0}(t)$

was regressed on the sum of the first three subharmonics of the 11-year cycle and of the Gleissberg cycle (8th subharmonic) mentioned earlier:

$$
\begin{aligned}
c_{r}(t) & =\sum_{N}\left[a_{N} \sin \left(\frac{2 \pi}{11.1 N} t\right)+b_{N} \cos \left(\frac{2 \pi}{11.1 N} t\right)\right]+k, \\
N & =2,3,4,8 .
\end{aligned}
$$

The reason for choosing this set of $N$-values is that the first three subharmonics have been found, via Fourier analysis, in at least two widely separated terrestrial occurrences in which the solar factor is found implicated
(Clark, 1988; Williams, et al. 1985). The eighth subharmonic was added heuristically, because its inclusion was found to triple the value of the regression's goodness-offit coefficient $R^{2}$. This subharmonic is, of course, the Gleissberg cycle already mentioned.

The $a_{N}, b_{N}$ values found through regression are shown to be:

$\begin{array}{llll}\text { coef. } \times 10^{6} & S_{\mathrm{I}} & S_{\text {II }} & S_{\text {III }} \\ a_{2} & 0.397(0.043) & -7.348(0.033) & -1.766(0.049) \\ b_{2} & 0.441(0.074) & 14.815(0.088) & 0.385(0.029) \\ a_{3} & 0.897(0.061) & -24.096(0.077) & -1.294(0.059) \\ b_{3} & 0.506(0.055) & -0.197(0.068) & -1.817(0.070) \\ a_{4} & 1.732(0.071) & -8.602(0.081) & 2.808(0.091) \\ b_{4} & -2.384(0.067) & 5.618(0.077) & 2.843(0.087) \\ a_{8} & 7.850(0.059) & 4.271(0.090) & -0.373(0.092) \\ b_{8} & 0.601(0.078) & 11.552(0.045) & -0.084(0.009) \\ k & 0.136 & 3.093 & 0.855 \\ R^{2} & 0.902 & 0.789 & 0.758 .\end{array}$

Figure 5 shows the complete model $\bar{z}_{11}(t)$. It is worth noticing the similarity between $\bar{z}_{11}(t)$ and J.A. Eddy's annual mean sunspot numbers at maxima in the 11year cycle (Eddy, 1976, Fig. 8). The degree of agreement between actual and modeled surge may be judged based upon the mean and standard deviation of the two:

\begin{tabular}{lllllll} 
& \multicolumn{1}{c}{$S_{\text {I }}$} & & $S_{\text {II }}$ & & $S_{\text {III }}$ & \\
& actual & model & actual & model & actual & model \\
avg & 32.33 & 32.51 & 21.26 & 21.29 & 32.21 & 32.98 \\
std & 16.60 & 16.66 & 15.01 & 14.40 & 18.94 & 20.90.
\end{tabular}

Before leaving the topic of the long-term $z_{11}(t)$ surging of solar activity, we may point out the fact that the trigger-like nature of the "mediating factor" is akin to 


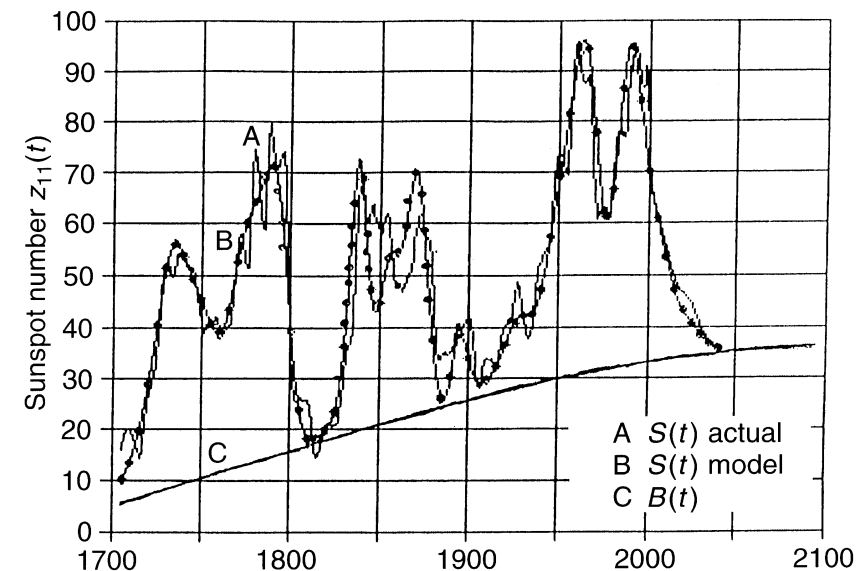

Fig. 5. Surges (actual and model)

the "eruption mechanism" that has been postulated for the 11-year sunspot cycle $z(t)$ itself (Kiepenheuer, 1959). As Dicke puts it, "It has become clear by now that the rise and fall in the number of spots is due to a number of practically independent individual processes: thus the idea of a true periodic phenomenon was dropped in favor of the so-called "eruption hypothesis" (Dicke, 1978).

\subsection{The derivative $D z_{11}(t)$}

As in most dynamic processes, the time-derivative $D z_{11}(t)$ of $z_{11}(t)$ can be instrumental in cases where $z_{11}(t)$ is a casual factor for the process at hand. It can be computed either from Eq. (1) or from the first difference of $z_{11}(t)$ as a surrogate which, according to Eq. (7), results in:

$z(t)=11 D z_{11}(t)+z(t-11)$,

and offers the means of projecting the sunspot number 11 years ahead.

Since Eq. (18) makes possible the extension of the model $z_{11}(t)$ beyond the present, a projection is possible with an accuracy commensurate with the fidelity of the

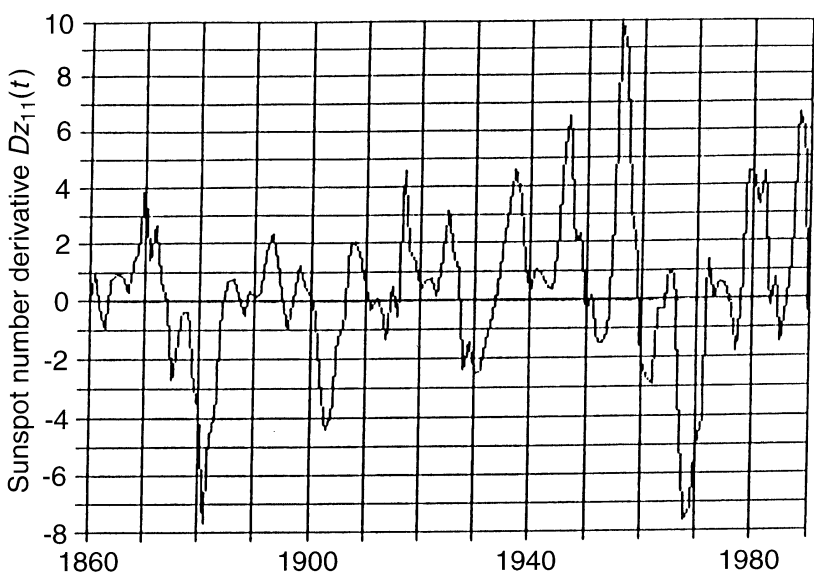

Fig. 6. Long-term sunspot derivative model $z_{11}(t)$; Fig. 6 shows the latter, revealing a regularity, which we discuss further later in this study.

\section{Model implementation}

The analytical means through which the present model is to be applied in seeking possible links between longterm solar activity on the one hand, and terrestrial phenomena on the other, range from the simple to the fairly sophisticated. In the sense that this means "connecting" one function of time $f(t)$ with another $g(t)$, the simplest relationship will be the direct one:

$$
\begin{gathered}
g(t)=a f(t)+b \\
a, b=\text { const. }
\end{gathered}
$$

or, when the data ranges warrant it,

$$
g_{N}(t)=\frac{g(t)-\bar{g}}{\sigma_{g}}=a \frac{f(t)-\bar{f}}{\sigma_{f}}+b,
$$

where $\bar{g}$ and $\bar{f}$ are the averages, $\sigma_{g}$ and $\sigma_{f}$ the standard deviations of the two functions, and $g_{N}(t), f_{N}(t)$ the normalized $g(t)$ and $f(t)$. This simple transformation brings the two data ranges within common bounds, and when necessary, can be rendered more effective if before normalization, $g(t)$ were "stretched" to:

$g_{\infty}(t)=g(t)^{m}$.

Normalization allows for a meaningful quantitative comparison of the contribution of multiple inputs $f_{1}(t) \ldots f_{n}(t)$ affecting a common output $g(t)$. Normalization, therefore, will be applied routinely in the entries of Part II of the study.

Where a more complex dynamic input-output connection is involved or suspected than that of Eq. (27), the powerful Kolmogorov method of signal-processing and system-response may be applied to advantage (Kolmogorov, 1941). Signal-processing pertains to either the interpolation or extrapolation of $f(t)$, while system-response addresses underlying causality or coevolution; generally, both cases have to cope with contamination from extraneous noise. The method has proved most effective in signal detection and estimation, as well as in control system analysis and design. A simplified derivation of the method by this author is given in the following.

Kolmogorov's formulation of the signal extrapolation case assumes that a function $f(t)$ of $t$, where both $t$ and $f(t)$ are real variables, is given in terms of equally spaced samples

$f(-n), f(-n+1), \ldots f(-1), f(0)$,

$f(0)$ being the current value, and that its value $f(k), k$ units of time into the future, is to be predicted. It is assumed that $f(t)$ is weakly stationary and, therefore,

1. that it has a finite second moment

$E\left[f(t)^{2}\right]<\infty$ 
where $E$ stands for expected value;

2. that the joint probability of any two of its values is a function of their time separation only:

$E[f(t) f(t+\tau)]=Q_{\mathrm{ff}}(\tau)$

3. that it has a continuous covariance function $Q_{\mathrm{ff}}(\tau)$.

Assuming that all information available on $f(t)$ is contained in the already-known samples means that the simplest way of finding its value $f(k) k$ time-units into the future will be to express the latter as a linear combination of the former:

$f(k)=\sum_{-n}^{0} a_{i} f(i)$.

Given that, by definition, the variance $\sigma^{2}$ of the error between the true value of $f(k)$ and its serial representation is:

$\sigma^{2}=E\left[f(k)-\sum_{-n}^{0} a_{i} f(i)\right]^{2}$,

minimization of $\sigma^{2}$ requires that its first $n+1$ derivatives with respect to $a_{i}$ be equated to zero. This results in $n+1$ equations in the unknowns $a_{i}$,

$E[f(k) f(0)]=a_{0} E[f(0) f(0)]+\cdots+a_{n} E[f(-n) f(0)]$,

$E[f(k) f(-1)]=a_{0} E[f(0) f(-1)]+\cdots+a_{n} E[f(-n) f(-1)]$,

$E[f(k) f(-n)]=a_{0} E[f(0) f(-n)]+\cdots+a_{n}[f(-n) f(-n)]$.

However, because of the weak stationarity condition, Eq. (34),

$E[f(k) f(k-\tau)]=E[f(t) f(t-\tau)]=Q_{\mathrm{ff}}(-\tau)$.

Furthermore, since $f(t)$ is a real quantity, its autocovariance function $Q_{\mathrm{ff}}$ has to be an even function:

$Q_{\mathrm{ff}}(-\tau)=Q_{\mathrm{ff}}(+\tau)$.

Thus, the system of Eqs. (35) becomes:

$$
\begin{aligned}
& \left|\begin{array}{ccccc}
Q_{\mathrm{ff}}(0) & Q_{\mathrm{ff}}(1) & Q_{\mathrm{ff}}(2) & \ldots & Q_{\mathrm{ff}}(n) \\
Q_{\mathrm{ff}}(1) & Q_{\mathrm{ff}}(0) & Q_{\mathrm{ff}}(1) & \ldots & Q_{\mathrm{ff}}(n-1) \\
\vdots & \vdots & \vdots & & \vdots \\
Q_{\mathrm{ff}}(n) & Q_{\mathrm{ff}}(n-1) & \ldots & \ldots & Q_{\mathrm{ff}}(0)
\end{array}\right|\left|\begin{array}{c}
a_{0} \\
a_{1} \\
\vdots \\
a_{n}
\end{array}\right| \\
& \quad=\left|\begin{array}{c}
Q_{\mathrm{ff}}(k) \\
Q_{\mathrm{ff}}(k+1) \\
\vdots \\
Q_{\mathrm{ff}}(k+n)
\end{array}\right|
\end{aligned}
$$

from which the unknowns $a_{i}$ can be computed.

While the analysis that led to Eq. (38) is based on the unit-time step, the unit's length has not been specified: it can be one year, one decade, or any other convenient magnitude. The potential of this tool in projecting $D z_{11}(t)$ into the future is obvious.
Besides the case of signal extrapolation just seen, the Kolmogorov method can be used in the problem of system-response as well. In this case an output or response function is supposed to be the result of an input or forcing function being modified by a linear operator. In the present context, $g(t)$, for instance, may be the result of the atmosphere's or hydrosphere's intervening between energy inputs from the sun and an ensuing climatic or other effect. Here again the representation:

$g(0)=\sum_{-n}^{0} a_{i} f(i)$

is used.

Comparison with Eq. (33) shows that the solution given by Eq. (30) will be the result, except for the fact that the output vector in the right-hand side of Eq. (38) is now replaced by the vector:

$\left[Q_{\mathrm{fg}}(0), Q_{\mathrm{fg}}(-1), \ldots Q_{\mathrm{fg}}(-n)\right]^{\mathrm{T}}$,

$Q_{\mathrm{fg}}($.$) being the cross-correlation function between f(t)$ and $g(t)$.

In the case of continuous time-functions the response relationship between output $g(t)$ and input $f(t)$ is given by the expression:

$g(0)=\int_{-t}^{0} f(t) w(t) \mathrm{d} t$,

where $w(t)$ is the transfer function of the process (James et al., 1947).

For the case of a discrete functional representation where both $f(t)$ and $g(t)$ are given in terms of equally spaced samples, Eq. (41) has the counterpart:

$g(0)=\sum_{-n}^{0} f(i) w(i)$

Comparison of Eq. (41) with Eq. (34) shows that:

$$
\begin{aligned}
& a_{i}=w(i) \\
& \quad i=0,1,2, \ldots,
\end{aligned}
$$

meaning that the vector $\left[a_{0}, a_{-1}, \ldots a_{-n}\right]^{\mathrm{T}}$ is the system response (or transfer) function, between $f(t)$ and $g(t)$ in the serial case. In the present context, of course, $f(t)=z_{11}(t)$.

Table 3 lists the values of $Q_{\mathrm{ff}}($.$) for z(t), z_{11}(t)$, and $D z_{11}(t)$, and Fig. 7 portrays the outcome. In the first curve the 11-year cycle of the sunspot number dominates; in the second curve one half of the Gleissberg cycle is obvious, and in the third a new and unexpected oscillation whose period is

$t_{a}=8.25$ years

manifests itself. This is a vivid example of nonlinear systems' "propensity to lapse into oscillations of new and incommensurable frequencies" (McDonald, 1980), and has been previously detected in sunspot periodograms based on maximum entropy spectral analysis (Cohen et al., 1974). 
Table 3. Autocorrelation functions (note: the correlations $Q_{\mathrm{ff}}$ are for the functions $f(t)-f_{a v}$, where $[z]_{a v}=59.25$, $\left[z_{11}\right]_{a v}=48.61$, $\left[D z_{11}\right]_{a v}=2.97$; the time-spans are $1890-1990$ for $z(t), 1755-1990$ for $z_{11}(t)$, and $1740-1990$ for $\left.D z_{11}(t)\right)$

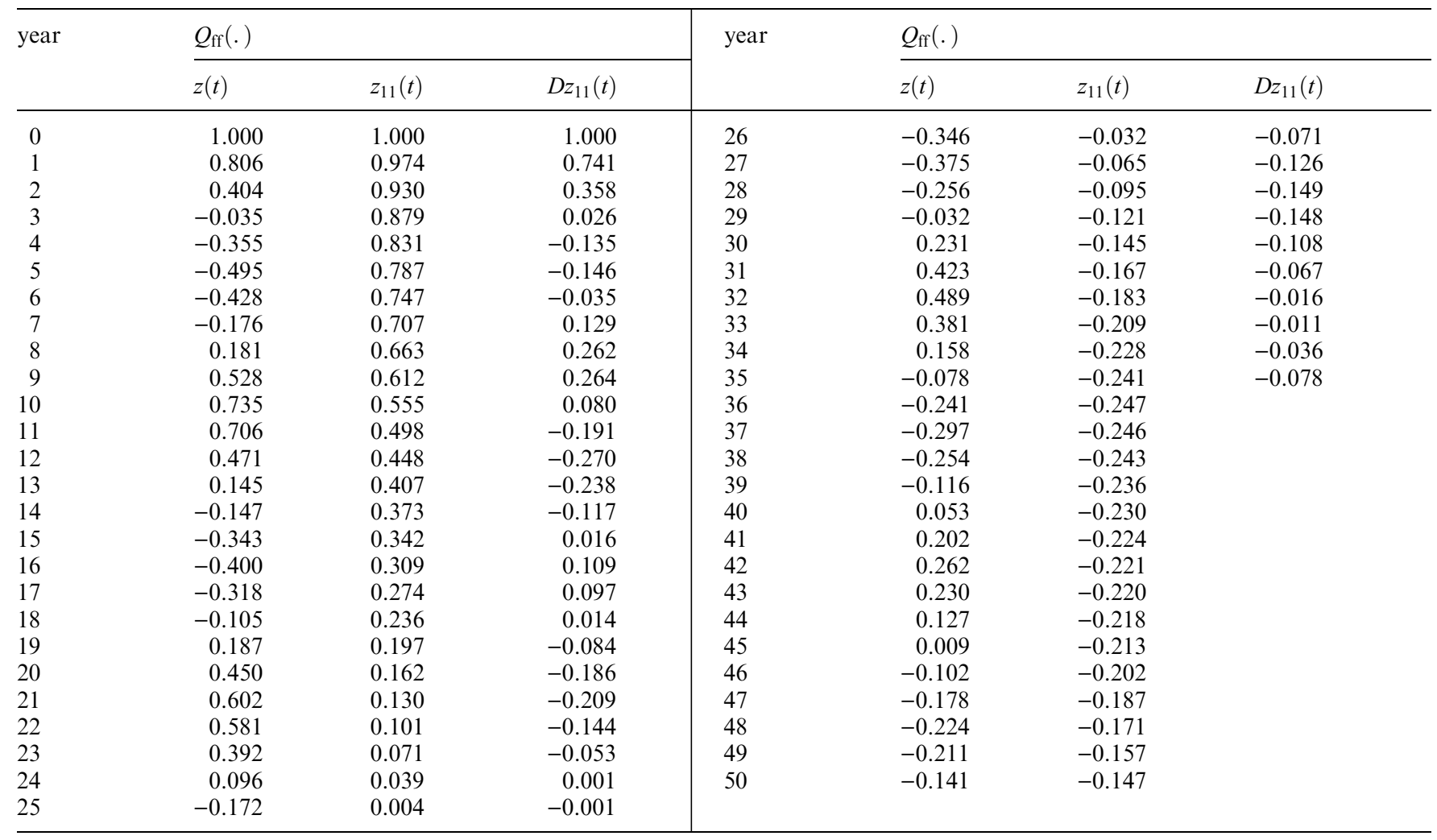

One feature of the Kolmogorov algorithm as presented here is that its definition in Eqs. (33) and (39) does not provide for a constant term, having been developed originally in a communication-signal context. Care, therefore, needs to be exercized when applied to signals from other realms.

Equally challenging in the context of the present theme is the pertinence of the cross-correlation function $Q f g($.$) : it has to do with the case where the cause side of$ the presumed cause/effect relationship $g(t)=$ function of $f(t)$ per diagram A:

$$
f(t) \longrightarrow g(t)
$$

is actually more complex as in diagram $\mathrm{B}$,

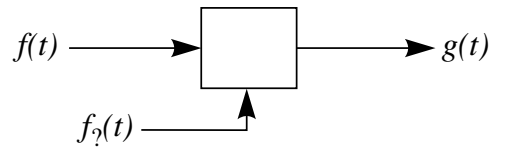

but the factor $f_{?}(t)$ has been omitted for lack of knowledge regarding the innards of the phenomenon at hand. In this circumstance application of the Kolmogorov algorithm on the system A instead of B will leave a residual in which the presence of $f_{?}(t)$ remains to be detected and extracted. This of course can be a difficult task, especially when incommensurable frequencies enter the picture. A case in point is terrestrial climate where not only the energy inputed by the sun is a factor, but contaminants, either manmade (Plantico et al., 1990) or subterranean (Schneider and Mass, 1975;
Lamb, 1970), which are released into the atmosphere play a significant role. Only in the case of known factors $f(t), f_{?}(t)$ acting additively can the algorithm in Eq. (45) be changed explicitly to represent the case.

For the record, Table 4 gives the actual and model values of the long-term solar activity's surrogate $z_{11}(t)$.

\section{Sunspot number projection}

We conclude Part I of this essay by demonstrating the model's ability to effect an 11-year projection of the annual sunspot number itself over the past 150 years; this via Eq. (33).

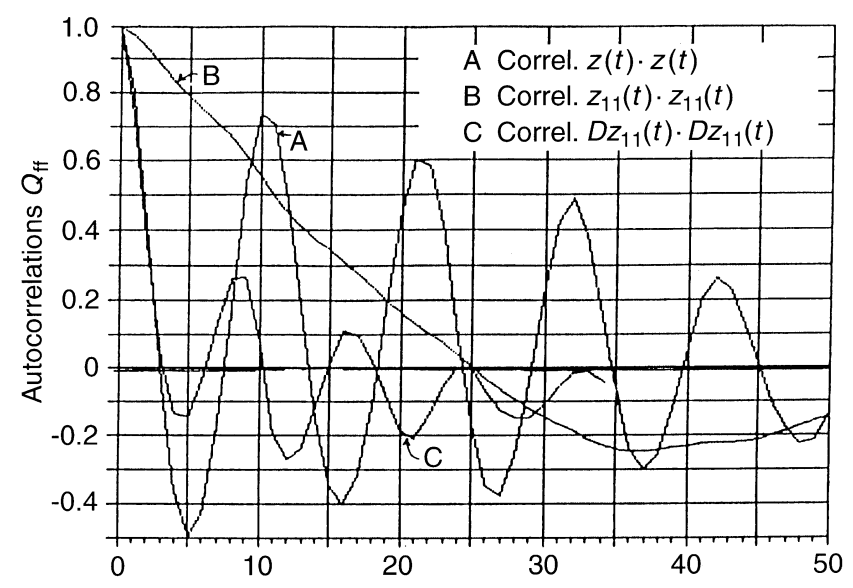

Fig. 7. Component autocovariances 
Table 4. Long-term solar activity $z_{11}(t)$

\begin{tabular}{|c|c|c|c|c|c|c|c|c|c|}
\hline year & $z_{11}$ & year & $z_{11}$ & year & $z_{11}$ & year & $z_{11}$ & year & $z_{11}$ \\
\hline 1790 & 72.94 & 1830 & 31.01 & 1870 & 57.07 & 1910 & 33.99 & 1950 & 73.1 \\
\hline 1791 & 71.28 & 1831 & 33.94 & 1871 & 58.47 & 1911 & 33.65 & 1951 & 73.25 \\
\hline 1793 & 71.31 & 1833 & 36.25 & 1873 & 61.79 & 1913 & 33.4 & 1953 & 70.27 \\
\hline 1794 & 72.96 & 1834 & 37.28 & 1874 & 61.85 & 1914 & 32.05 & 1954 & 69.19 \\
\hline 1795 & 73.97 & 1835 & 41.68 & 1875 & 59.13 & 1915 & 32.55 & 1955 & 71.77 \\
\hline 1796 & 73.24 & 1836 & 51.22 & 1876 & 57.38 & 1916 & 31.96 & 1956 & 81.64 \\
\hline 1797 & 66.28 & 1837 & 60.49 & 1877 & 57.03 & 1917 & 36.52 & 1957 & 90.51 \\
\hline 1798 & 54.65 & 1838 & 65.27 & 1878 & 56.67 & 1918 & 38.21 & 1958 & 93.53 \\
\hline 1799 & 43.37 & 1839 & 67.23 & 1879 & 53.8 & 1919 & 39.58 & 1959 & 95.59 \\
\hline 1800 & 33.95 & 1840 & 67.01 & 1880 & 50.01 & 1920 & 39.92 & 1960 & 93.55 \\
\hline 1801 & 28.87 & 1841 & 63.9 & 1881 & 42.31 & 1921 & 40.6 & 1961 & 90.83 \\
\hline 1802 & 26.91 & 1842 & 61.75 & 1882 & 37.62 & 1922 & 41.37 & 1962 & 87.93 \\
\hline 1803 & 25.37 & 1843 & 60.23 & 1883 & 33.72 & 1923 & 41.57 & 1963 & 87.6 \\
\hline 1804 & 25.43 & 1844 & 60.82 & 1884 & 33.47 & 1924 & 42.96 & 1964 & 87.26 \\
\hline 1806 & 26.15 & 1846 & 63.68 & 1886 & 34.92 & 1926 & 47.62 & 1966 & 89.05 \\
\hline 1807 & 25.62 & 1847 & 61.59 & 1887 & 35.08 & 1927 & 48.7 & 1967 & 84.7 \\
\hline 1808 & 25.81 & 1848 & 60.35 & 1888 & 34.57 & 1928 & 46.33 & 1968 & 77.04 \\
\hline 1809 & 25.66 & 1849 & 59.82 & 1889 & 34.93 & 1929 & 44.9 & 1969 & 69.83 \\
\hline 1810 & 25.05 & 1850 & 58.08 & 1890 & 35.03 & 1930 & 42.36 & 1970 & 64.87 \\
\hline 1811 & 23.86 & 1851 & 58.07 & 1891 & 35.33 & 1931 & 39.96 & 1971 & 60.72 \\
\hline 1812 & 21.23 & 1852 & 59.65 & 1892 & 37.03 & 1932 & 38.6 & 1972 & 62.08 \\
\hline 1813 & 18.25 & 1853 & 61 & 1893 & 39.35 & 1933 & 37.83 & 1973 & 62.13 \\
\hline 1814 & 15.59 & 1854 & 61.9 & 1894 & 40.65 & 1934 & 38.09 & 1974 & 62.73 \\
\hline 1815 & 14.49 & 1855 & 61.15 & 1895 & 40.69 & 1935 & 39.85 & 1975 & 63.21 \\
\hline 1816 & 14.82 & 1856 & 57.89 & 1896 & 39.75 & 1936 & 43.07 & 1976 & 62.98 \\
\hline 1817 & 16 & 1857 & 54.36 & 1897 & 39.82 & 1937 & 47.66 & 1977 & 61.21 \\
\hline 1818 & 17.82 & 1858 & 50.39 & 1898 & 41.05 & 1938 & 51.35 & 1978 & 61.09 \\
\hline 1819 & 19.22 & 1859 & 47.58 & 1899 & 41.54 & 1939 & 52.35 & 1979 & 65.6 \\
\hline 1820 & 20.41 & 1860 & 47.54 & 1900 & 41.74 & 1940 & 52.62 & 1980 & 70.07 \\
\hline 1821 & 21 & 1861 & 48.5 & 1901 & 41.34 & 1941 & 53.69 & 1981 & 73.35 \\
\hline 1825 & 20.85 & 1865 & 48.72 & 1905 & 28.9 & 1945 & 57.6 & 1985 & 76.83 \\
\hline 1826 & 20.94 & 1866 & 49.59 & 1906 & 27.97 & 1946 & 62.74 & 1986 & 76.64 \\
\hline 1827 & 21.28 & 1867 & 49.86 & 1907 & 29.81 & 1947 & 69.27 & 1987 & 78.15 \\
\hline 1828 & 23.38 & 1868 & 51.22 & 1908 & 31.84 & 1948 & 71.26 & 1988 & 84.75 \\
\hline \multirow[t]{2}{*}{1829} & 26.74 & 1869 & 52.96 & 1909 & 33.4 & 1949 & 73.55 & 1989 & 90.68 \\
\hline & & & & & & & & 1990 & 89.5 \\
\hline
\end{tabular}

A reliable prediction of the 11-year cycle "could be of great importance to many fields of human activity; telecommunications, space exploration, and climate forecasting being among them" (Fyodorof et al., 1996). Over the years the list of attempts to produce a solution has been long: see for instance, Wilson (1985), McNish and Lincoln (1987), Withbroe (1989), and FriisChristensen and Lassen (1991). Although the descriptive schemes have been many, and the numerical models range from simple to fairly elaborate, none has achieved the goal, including those based on a Fourier transform. The disappointment is due, of course, to the limited knowledge of the dynamics of the astrophysical process taking place. It is the view of this study that the Kolmogorov algorithm can help with the quest, and produce a better descriptor. For this, the suggested modus operandi proceeds in three successive steps:

1. Generate an 11-year Kolmogorov projection $z_{a}(t)$ of $z(t)$ based on three past equidistant states of it: $z(t-33), z(t-22)$, and $z(t-11)$.
2. Search the residual $r(t)=z(t)-z_{a}(t)$ for the presence of both the basic 11-year solar cycle and the longterm $z_{11}(t)$ or its derivative .

3 . Finally, apply regression analysis to the residual $r(t)$ as a function of the terms found in Step 2.

The first step requires solving the system:

$\left|\begin{array}{lll}Q(0) & Q(11) & Q(22) \\ Q(11) & Q(0) & Q(11) \\ Q(22) & Q(11) & Q(0)\end{array}\right|=\left|\begin{array}{l}a_{11} \\ a_{22} \\ a_{33}\end{array}\right|\left|\begin{array}{l}Q(11) \\ Q(22) \\ Q(33)\end{array}\right|$,

from which, based on Table 3 for the values of $Q($.$) of$ the autocorrelation of $z(t)$, the following transfer function is computed:

$$
\begin{array}{cc}
i & W(i) \\
-33 & -0.00179 \\
-22 & +0.25351 \\
-11 & +0.97182
\end{array}
$$

effecting the projection: 
$z_{a}(t)=\sum_{i} z(t-i)$

Since $z_{a}(t)$ and $z(t)$ have a concurrent part, the former can be improved by making it:

$z_{a}(t)=m z(t)+n$

and finding the parameters $a, b$ through linear regression:

$$
\begin{aligned}
& m=0.855(0.067), \\
& n=15.270, \\
& R^{2}=0.532 .
\end{aligned}
$$

This step serves to compensate for the absence of a constant term in the definition of the Kolmogorov algorithm mentioned earlier.

In the residual $r(t)$ of the operation described by Eq. (56), the presence of $D z_{11}$ is discerned, and in the same residual's autocorrelation function (Fig. 8) the presence of the sunspot number's basic 11-year cycle is obvious. Hence, $r(t)$ is set as:

$r(t)=a \sin (2 \pi / 11 * t)+b \cos (2 \pi / 11 * t)+c D z_{11}(t)+d$,

from which a new regression produces:

$$
\begin{aligned}
& a=-9.957(0.755), \\
& b=-0.867(0.755), \\
& c=9.802(0.186), \\
& d=-2.326, \\
& R^{2}=0.949, \\
& t_{0}=1830 .
\end{aligned}
$$

Thus, the complete 11-year projection expression becomes:

$z(t+11)=z_{a}(t)+r(t+11)$.

In this expression the $D z_{11}(t+11)$ term present in $r(t+11)$ comes from the actual $z_{11}(t)$ up to the year 1995 and the model $z_{11}(t)$ beyond that.

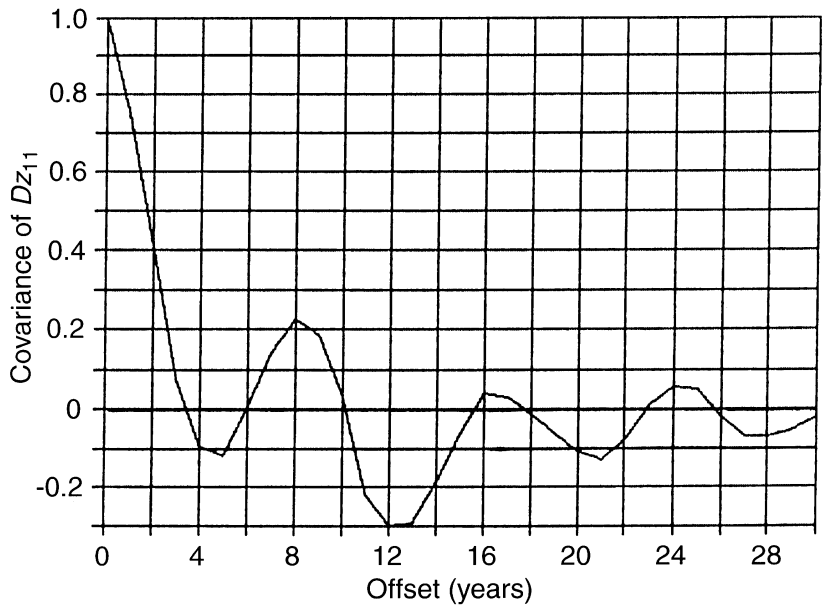

Fig. 8. Autocovariance of long-term derivative

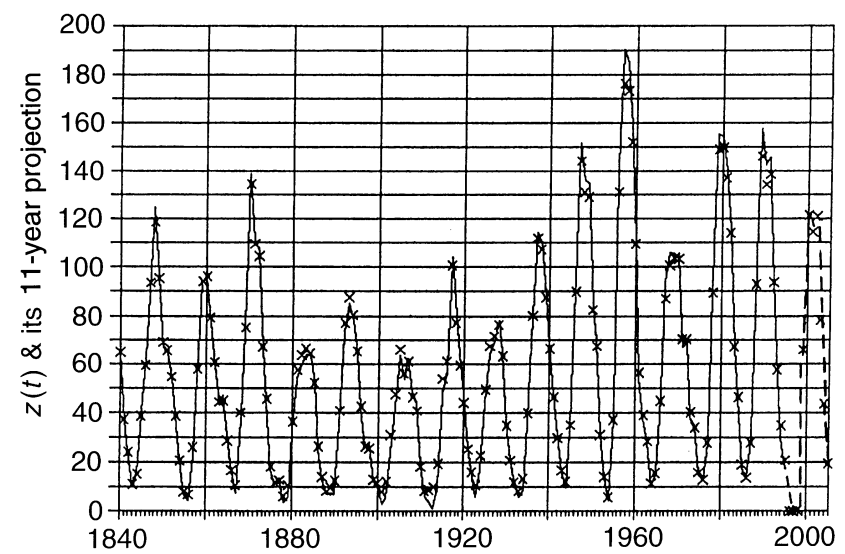

Fig. 9. Sunspot number 11-year projection

The final outcome may be seen in Fig. 9, while Table 5 lists the 1990- 2005 projections. The strength of the approach can be assessed by the fact that in the 1835-1995 record of fifteen $z(t)$ maxima and fourteen minima, the model missed only the 1968 maximum by 1 year and the 1913 minimum by 2 years, which is smaller than the 2.3 years suggested by Fyodorov et al. (1996) as the limit for useful prediction.

Equally important is the fact that over the same timespan the Kolmogorov term of the 11-year prediction captured $86 \%$ of the $z(t)$ signal; this is based on the ratio of the standard deviation (37.6) of $z(t)$-predicted versus (41.5) of $z(t)$-actual. For the complete prediction algorithm the ratio is 0.94 . As far as the sunspot number's peak values are concerned, the projection error is given in Table 6. The approach may be compared to the

Table 5. $z(t)$ 11-year projection

\begin{tabular}{llc|cc}
\hline year & \multicolumn{1}{l|}{$z(t)$} & year & $z(t)$ \\
\cline { 2 - 3 } & actual & projected & & projected \\
\hline 1990 & 142.65 & 127.6 & 1999 & 53.4 \\
1991 & 145.2 & 134.6 & 2000 & 111.9 \\
1992 & 94.3 & 91.0 & 2001 & 107.9 \\
1993 & 54.5 & 53.9 & 2002 & 116.8 \\
1994 & 29.9 & 29.1 & 2003 & 75.1 \\
1995 & 17.5 & 12.4 & 2004 & 40.0 \\
1996 & & 8.0 & 2005 & 14.1 \\
1997 & & 0 & 2006 & 0 \\
1998 & & 0 & & \\
\hline
\end{tabular}

Table 6. Peak projection error

\begin{tabular}{ll|ll}
\hline cycle no. & error $\%$ & cycle no. & error $\%$ \\
\hline 9 & +4.7 & 16 & +2.1 \\
10 & +1.7 & 17 & +2.1 \\
11 & +1.3 & 18 & +1.5 \\
12 & -3.6 & 19 & +8.6 \\
13 & -2.8 & 20 & +2.1 \\
14 & -3.9 & 21 & +3.7 \\
15 & +3.1 & 22 & +7.2 \\
\hline
\end{tabular}


present lean state of the sunspot prediction art as conducted at

the 1996 NASA "consensus panel" convened under Jo Ann Joselyn of the National Oceanic and Atmospheric Administration for the purpose of predicting the maximum of the next sunspot cycle: the panel considered 28 prediction methods of all kinds including numerology-based ones - and settled on a maximum for the upcoming sunspot cycle no. 23 equal to that of the ongoing 22 nd one. Considering that sunspot peaks are accompanied by the emission of highly energetic particles and magnetic storms that can blast the delicate electronics of military, scientific, and commercial satellites, and that the ultraviolet radiation from an active sun heats up Earth's upper atmosphere causing it to expand and exert extra drag on satellites, a real barn-burner of a maximum could create the need of new shuttle missions to reboost the orbiting Hubble Space Telescope and the planned space station" (Glanz, 1997).

\section{Conclusions}

The analysis detailed in the foregoing sections brought out and modeled three main features of long-term solar activity based on the sunspot number as the activity's surrogate: a rising trend, long-term surging, and a patterned transient. Although the idea of using the sunspot number to gauge solar activity is not new (Brown, 1974), the casting of this activity in the role of a long-term dynamic surging agent is. Noteworthy is the fact that the model points to the present decade being the time of peaking of the latest surge $S_{\text {III }}$ of long-term solar activity to date, after which a span of roughly five decades of gradual subsiding is to follow. It is also significant that the macrodynamics of a phenomenon as complex as the energy exchanges taking place in the sun's interior can be portrayed in terms of a representation as simple and compact as the mediating factor having the characteristics of a trigger mechanism. This concept, coupled with the system-response tool of the Kolmogorov formulation, make for an effective analytical tool for the study of at least the phenomenology of sun-related long-term terrestrial phenomena, as Part II of this study will demonstrate. It should be pointed out that the model's synthesis made use of long-term periodicities present in particular geophysical topics where solar energy certainly has a role. Beyond this, the mediating factor may, as an operational concept, have a bearing upon the search for the modus operandi of the complex dynamics of the interior of the sun itself (Cox et al., 1993). Finally, particular attention of the Kolmogorov algorithm by the applied scientist in any field is warranted; this because of its premise that the dynamics of the studied phenomenon is contained in the historical data itself, even if no theory exists about such dynamics at the present time.

Acknowledgements. The Editor-in-chief thanks two referees for their help in evaluating this paper.

\section{References}

Brown, G. N., A new solar-terrestrial relationship, Nature, 251, 592-594, 1974.

Clark, J. S., The effect of climate change in fire regimes in northwestern Minnesota, Nature, 334, 233-235, 1988.

Cohen, T. J., and P. R. Lintz, Long-term periodicities in the Sun, Nature, 250, 398-399, 1974.

Cox, A. N., W. Livingston, and M. Matthews (Eds.), Solar interior and atmosphere, Arizona University Press, Tucson, 1993.

Dicke, R. K., Is there a chronometer hidden deep in the Sun? Nature, 276, 676-680, 1978.

Eddy, J. A., The Maunder minimum, Science, 192, 1189-1202, 1976.

Eddy, J. A., and M. Stuiver, The case of the missing sunspots, Sci. Am., May, 88-92, 1977.

Friis-Christensen, E., and K. Lassen, Length of the solar cycle: an indicator of solar activity closely associated with climate, Science, 254, 698-700, 1991.

Fyodorov, M. V., V. V. Klimenko, and V. V. Dovgalyuk, Sunspot minima dates: a secular forecast, Solar Phys., 165, 193-99, 1996.

Glanz, J., Bold prediction downplays the Sun's next peak, Science, 275, 927, 1997.

Gribbin, J. R., and S. Plagemann, The Jupiter effect, Walker, New York, 1974.

Hoyt, D. V., K. H. Schatten, and E. Nesmes-Ribes, The one hundreth year of Rudolf Wolf's death: do we have the correct reconstruction of solar activity? Geophys. Res. Lett., 21, 20672070, 1994.

Hughes, D. W., The inconsistent Sun, Nature, 266, 405-406, 1977.

James, H. M., N. R. Nichols, and R. S. Phillips (Eds.), Theory of servomechanisms, McGraw Hill, New York, 1947.

Jose, P. D., Sun's motion and sunspots, Astron. J., 70, 193-200, 1965.

Keyfitz, N., Science fragmented, Options Int. Inst. Adv. Syst. Anal., $5-12,1992$.

Keyfitz, N., On the secterianism of science, Options Int. Inst. Adv. Syst. Anal., 1-2, 1994.

Kiepenheuer, K., The Sun, University of Michigan Press, Ann Arbor, 1959.

Kolmogorov, A. N., Interpolation und Extrapolation von stationären zufälligen Folgen, Bull. Acad. Sci. U.S.S.R., Ser. Math. 5, 1941

Lamb, H. H. Volcanic dust in the atmosphere: with a chronology and assessment of its meteorological significance, Phil. Trans. R. Soc. Lond., 266, 425-527, 1970.

Lotka, A. J., Elements of mathematical biology, Dover, New York, 1956.

May, R. M., Simple mathematical models with very complicated dynamics, Nature, 261, 474-484, 1976.

McDonald, M., Nonlinear dynamics, Nature, 283, 431- 432, 1980.

McKinnon, J. A., Sunspot numbers: 1610-1985, Rep. UAG-95, World Data Center A, National Academy of Sciences, Washington DC, 1987.

McNish, A. G., and J. V. Lincoln, Table of observed and predicted relative sunspot numbers and solar flux, Solar Geophys. Data Suppl., 9-14, 1987.

Modis, T., and A. Bebecker, Chaos-like states can be expected before and after a logistic growth. Technol. Forecast. Social Change, 41, 111-120, 1992.

Mullin, T., and T. J. Price, Experimental observation of chaos arising from the interaction of steady and time-dependent flows, Nature, 340, 294-296, 1989.

Plantico, M. S., T. R. Karl, G. Kukla, and J. Gavin, Is recent climate change across the United States related to rising levels of anthropogenic greenhouse gasses? J. Geophys. Res., 95, 16617-16637, 1990.

Samuel, Sir H. L., Essay in physics, Harcourt, New York, 1952. 
Schneider, S. M., and C. Mass Volcanic dust, sunspots, and temperature trends, Science, 190, 741-746, 1975.

Stuiver, M., and P. D. Quay, Changes in atmospheric carbon-14 attributed to a variable sun, Science, 207, 11-19, 1980.

Williams, G. E., Sunspot periods in the late Precambrian glacial climate and solar-planetary relations, Nature, 291, 624-628, 1981.

Williams, G. E., and C. P. Sonnett, Solar signature in sedimentary cycles from the Elatina formation, Australia, Nature, 318, 523$527,1985$.
Wilson, R. M., On the use of "first spotless day" as a predictor for sunspot minimum, Solar Phys. 158, 197-204, 1985.

Withbroe, G. L., Solar activity cycle: history and prediction, $J$. Spacecraft, 26, 394-402, 1989.

Wood, K. D., Sunspots and planets, Nature, 240, 91-93, 1972. 